\title{
Strict Coincidence and Common Strict Fixed Point of Hybrid Pairs of Self-mappings with Application
}

\author{
Anita Tomar*, Shivangi Upadhyay and Ritu Sharma
}

(Communicated by Ishak ALTUN)

\begin{abstract}
In this paper, we discuss strict coincidence and common strict fixed point of strongly tangential hybrid pairs of self-mappings satisfying Kannan type contraction via $\delta$-distance, which is not even a metric. Also coincidence and common fixed point is established using Hausdorff metric. Consequently, several known results are extended, generalized and improved. Examples are given to illustrate our results and an application is also furnished to demonstrate the applicability of results obtained.
\end{abstract}

Keywords: Strict common fixed point, strict coincidence point, strongly tangential, hybrid pair.

AMS Subject Classification (2010): Primary: $47 \mathrm{H} 10$; Secondary: $54 \mathrm{H} 25$.

${ }^{*}$ Corresponding author

\section{Introduction}

The celebrated Banach contraction principle [1] is indeed a very significant result in metrical fixed point theory which states that a contraction mapping of a complete metric space into itself has a unique fixed point. It has several applications in solving nonlinear equations, but has one great disadvantage that contraction mapping forces involved mapping to be continuous throughout the metric space. The question of continuity of contractive mapping and in particular continuity at fixed points emerged with the publication of research papers by R. Kannan in 1968 and 1969 respectively ([7] and [8]).

A mapping T on a metric space $(X, d)$ is called Kannan contraction [7] if there exists $\alpha \in\left[0, \frac{1}{2}\right)$ such that

$$
d(T x, T y) \leq \alpha[d(x, T x)+d(y, T y)]
$$

for all $x, y \in X$. Let $X=\mathbb{R}$ be a usual metric space and $T: X \rightarrow X$ be a mapping defined by $T x=0$, if $x \in(-\infty, 2]$ and $T x=\frac{1}{2}$, if $x \in(2,+\infty)$. Then $T$ is not continuous on $\mathbb{R}$, but it satisfies Kannan contraction with $\alpha=\frac{1}{3}$ and has a fixed point at 0. In 1975, Subrahmanyam [13] proved that Kannan contraction characterizes the metric completeness. A metric space $(X, d)$ is complete if and only if every Kannan contraction on $X$ has a fixed point. On the other hand Markin [9] and Nadler [10] initiated the study of fixed points of multivalued mappings using the Hausdorff metric. Aim of this paper is to establish strict coincidence and common strict fixed point of strongly tangential hybrid pairs of self-mappings satisfying Kannan type contracion using $\delta$-distance, which is not even a metric. Also coincidence and common fixed point is established using Hausdorff metric. As an application results obtained are applied to establish the existence of solutions of functional equation arising in dynamic programming.

Received : 21-January-2017, Accepted : 27-May-2017 


\section{Preliminaries}

Throughout this paper, let $(X, d)$ be a metric space and $C B(X)$ be the family of all nonempty closed and bounded subsets of $X$.

Functions $\delta(A, B)$ and $D(A, B)$ are defined as:

$$
\delta(A, B)=\sup \{d(a, b): a \in A, b \in B\}
$$

and

$$
D(A, B)=\inf \{d(a, b): a \in A, b \in B\}
$$

for all $A, B \in C B(X)$. If $A=\{a\}$, then $\delta(A, B)=\delta(a, B)$. If $A=\{a\}$ and $B=\{b\}$, then $\delta(A, B)=d(a, b)$. It follows immediately from the definition of $\delta$ that

- $\delta(A, B)=\delta(B, A)>0$,

- $\delta(A, B) \leq \delta(A, C)+\delta(C, B)$,

- $\delta(A, B)=0$ iff $A=B=\{a\}$,

- $\delta(A, A)=\operatorname{diam} A$, for all $A, B, C \in C B(X)$.

Let $H$ be the Hausdorff metric with respect to $d$, i.e.,

$$
H(A, B)=\max \left\{\sup _{x \in A} d(x, B), \sup _{x \in B} d(x, A)\right\},
$$

where $d(x, A)=\inf \{d(x, y): y \in A\}$, for all $A \in C B(X)$. Also $H(A, B)=0$ iff $A=B$.

Let $f: X \rightarrow X$ be a single valued and $T: X \rightarrow C B(X)$ be a multivalued mapping of a metric space $(X, d)$. Then $(f, T)$ is called a hybrid pair of mapping.

For a multivalued mapping $T: X \rightarrow C B(X)$, a point $u \in X$ is

- fixed point if $u \in T u$.

- strict fixed point (or a stationary fixed point or absolute fixed point) if $T u=\{u\}$.

For a hybrid pair $(f, T)$, a point $u \in X$ is a

- coincidence point if $f u \in T u$;

- strict coincidence point if $T u=\{f u\}$;

- common fixed point if $u=f u \in T u$;

- common strict fixed point if $f u=T u=\{u\}$.

Definition 2.1. [6] A hybrid pair of mappings $(f, T)$ on a metric space $(X, d)$ is weakly commuting if $f T x \in C B(X)$ and $\delta(T f x, f T x) \leq \max \{\delta(f x, T x) ; \operatorname{diam}(f T x)\}$; for all $x \in X$.

Note that if $T$ is a single valued mapping, then the set $\{f T x\}$ consists of a single point. Therefore, $\operatorname{diamfTx}=0$ for all $x \in X$ and definition of weak commutativity of a hybrid pair of self mappings reduces to the weak commutativity of a single valued pair of self mappings given by Sessa [11], i.e., $d(T f x, f T x) \leq d(f x, T x)$, for all $x \in X$.

Definition 2.2. [12] A pair of single valued self mappings $(f, g)$ on a metric space $(X, d)$ is tangential with respect to a pair of multivalued self mappings $(S, T)$ if

$$
\lim _{n \rightarrow \infty} S x_{n}=\lim _{n \rightarrow \infty} T y_{n}=A \in C B(X),
$$

whenever $\left\{x_{n}\right\}$ and $\left\{y_{n}\right\}$ are sequences in $X$ such that

$$
\lim _{n \rightarrow \infty} f x_{n}=\lim _{n \rightarrow \infty} g y_{n}=z \in A
$$

for some $z \in X$. 
Definition 2.3. [4] A pair of single valued self mappings $(f, g)$ on a metric space $(X, d)$ is strongly tangential with respect to a pair of multivalued self mappings $(S, T)$ if

$$
\lim _{n \rightarrow \infty} S x_{n}=\lim _{n \rightarrow \infty} T y_{n}=A \in C B(X),
$$

whenever $\left\{x_{n}\right\}$ and $\left\{y_{n}\right\}$ are sequences in $X$ such that

$$
\lim _{n \rightarrow \infty} f x_{n}=\lim _{n \rightarrow \infty} g y_{n}=z \in A
$$

and $z \in f X \cap g X$.

Definition 2.4. [4] A single valued self mapping $f$ on a metric space $(X, d)$ is strongly tangential with respect to multivalued self mapping $T$ if

$$
\lim _{n \rightarrow \infty} T x_{n}=\lim _{n \rightarrow \infty} T y_{n}=A \in C B(X),
$$

whenever $\left\{x_{n}\right\}$ and $\left\{y_{n}\right\}$ are sequences in $X$ such that

$$
\lim _{n \rightarrow \infty} f x_{n}=\lim _{n \rightarrow \infty} f y_{n}=z \in A
$$

and $z \in f X$.

Definition 2.5. [5] Let $f: X \rightarrow X$ be a single valued mapping while $T: X \rightarrow C B(X)$ be a multivalued mapping. The mapping $f$ is said to be coincidentally idempotent with respect to mapping $T$, if $f x \in T x$ imply $f f x=f x$.

\section{Main results}

First we establish strict coincidence and common strict fixed point for two hybrid pairs of mappings satisfying Kannan type contraction using $\delta$-distance.

Theorem 3.1. Let $f, g: X \rightarrow X$ be single valued mappings and $S, T: X \rightarrow C B(X)$ be multi-valued mappings of metric space $(X, d)$. If there exists $\alpha \in\left[0, \frac{1}{2}\right)$ such that

$$
\delta(S x, T y) \leq \alpha[d(f x, S x)+d(g y, T y)], \forall x, y \in X
$$

and pair $(f, g)$ is strongly tangential with respect to $(S, T)$. Then pairs $(f, S)$ and $(g, T)$ have a strict coincidence point. Moreover, $f, g, S$ and $T$ have a unique common strict fixed point if hybrid pairs $(f, S)$ and $(g, T)$ are coincidentally idempotent.

Proof. Suppose that $(f, g)$ is strongly tangential with respect to $(S, T)$. Hence, there exist sequences $\left\{x_{n}\right\}$ and $\left\{y_{n}\right\}$ in $X$ such that $\lim _{n} f x_{n}=\lim _{n} g y_{n}=z \in A=\lim _{n} S x_{n}=\lim _{n} T y_{n}$, where $A \in C B(X)$ and $z \in f X \cap g X$. Hence, there exist $u$ and $v \in X$ such that $f u=g v=z$. Now we claim that $z=f u \in S u$, if not using $x=u$ and $y=y_{n}$ in condition (3.1),

$$
\delta\left(S u, T y_{n}\right) \leq \alpha\left[d(f u, S u)+d\left(g y_{n}, T y_{n}\right)\right] .
$$

Taking limit as $n \rightarrow \infty$, we get

$$
\delta(S u, A) \leq \alpha d(f u, S u)+\alpha d(z, A),
$$

or

$$
\delta(S u, A) \leq \alpha d(f u, S u) .
$$

But $f u=z \in A$. So,

$$
d(f u, S u) \leq \alpha d(f u, S u)<d(f u, S u),
$$

a contradiction. Therefore, $f u \in S u$, i.e., $\delta(S u, A)=0$, i.e., $S u=\{f u\}$. Hence, $f$ and $S$ have a strict coincidence point.

Now we claim that $z=g v \in T v$, if not using $x=x_{n}$ and $y=v$ in condition (3.1),

$$
\delta\left(S x_{n}, T v\right) \leq \alpha d\left(f x_{n}, S x_{n}\right)+\alpha d(g v, T v) .
$$

Taking limit as $n \rightarrow \infty$, we get

$$
\delta(A, T v) \leq \alpha d(z, A)+\alpha d(g v, T v)
$$


or

$$
\delta(A, T v) \leq \alpha d(g v, T v) .
$$

But $g v=z \in A$. So,

$$
d(g v, T v) \leq \alpha d(g v, T v)<d(g v, T v),
$$

a contradiction. Therefore, $g v \in T v$, i.e., $\delta(A, T v)=0$, i.e., $T v=\{g v\}$. Hence, $g$ and $T$ have a strict coincidence point. Hence $z \in S u=T v=\{z\}$. Now since $(f, S)$ is coincidentally idempotent, $f u \in S u$ implies $f f u=f u \in S u$. Now we claim that $z=f z \in S z$, if not using $x=z$ and $y=y_{n}$ in condition (3.1),

$$
\delta\left(S z, T y_{n}\right) \leq \alpha d(f z, S z)+\alpha d\left(g y_{n}, T y_{n}\right) .
$$

Taking limit as $n \rightarrow \infty$, we get

$$
\delta(S z, A) \leq \alpha d(f z, S z)+\alpha d(z, A),
$$

or

$$
\delta(S z, A) \leq \alpha d(f z, S z) .
$$

But $f z=z \in A$, So,

$$
d(f z, S z) \leq \alpha d(f z, S z)<d(f z, S z),
$$

a contradiction. Therefore, $f z \in S z$, i.e., $\delta(S z, A)=0$, i.e., $S z=\{f z=z\}$. Similarly, $(g, T)$ is coincidentally idempotent $g v \in T v$ implies $g g v=g v \in T v$.

Now we claim that $z=g z \in T z$, if not using $x=x_{n}$ and $y=z$ in condition (3.1),

$$
\delta\left(S x_{n}, T z\right) \leq \alpha d\left(f x_{n}, S x_{n}\right)+\alpha d(g z, T z) .
$$

Taking limit as $n \rightarrow \infty$, we get

$$
\delta(A, T z) \leq \alpha d(z, A)+\alpha d(g z, T z)
$$

or

$$
\delta(A, T z) \leq \alpha d(g z, T z)
$$

But $g z=z \in A$, i.e.,

$$
d(g z, T z) \leq \alpha d(g z, T z)<d(g z, T z)
$$

a contradiction. Therefore, $g z \in T z$, i.e., $\delta(A, T z)=0$ which implies $g z \in T z=\{g z=z\}$.Therefore $z$ is a common strict fixed point of $f, g, T$ and $S$.

Let $z$ and $w$ be two common strict fixed points such that $z \neq w$. Now from condition (3.1), we get

$$
\begin{gathered}
\delta(S z, T w) \leq \alpha[d(f z, S z)+d(g w, T w)] \\
\delta(S z, T w) \leq 0
\end{gathered}
$$

but

$$
\delta(S z, T w)>0,
$$

a contradiction. Hence, $z$ is a unique common strict fixed point of $f, g, T$ and $S$.

Taking $f=g$ and $T=S$ in Theorem 3.1, we obtain the following corollary:

Corollary 3.1. Let $f: X \rightarrow X$ be a single valued mapping and $T: X \rightarrow C B(X)$ be a multi-valued mapping of a metric space $(X, d)$. If there exists $\alpha \in\left[0, \frac{1}{2}\right)$ such that

$$
\delta(T x, T y) \leq \alpha[d(f x, T x)+d(f y, T y)], \forall x, y \in X
$$

and $f$ is strongly tangential with respect to $T$. Then $f$ and $T$ have a strict coincidence point. Moreover, $f$ and $T$ have a unique common strict fixed point if hybrid pair $(f, T)$ is coincidentally idempotent. 
Example 3.1. Let $X=[0,5]$ and $d$ be the usual metric on $X$. Let a hybrid pair of mappings $f$ and $T$ on $X$ be defined as follows:

$$
f x=\left\{\begin{array}{ll}
1-x, & 0 \leq x \leq \frac{1}{2} \\
5, & \frac{1}{2}<x \leq 5,
\end{array} \quad T x= \begin{cases}\left\{\frac{1}{2}\right\}, & 0 \leq x \leq \frac{1}{2} \\
{\left[1, \frac{3}{2}\right],} & \frac{1}{2}<x \leq 5 .\end{cases}\right.
$$

Consider the two sequences $\left\{x_{n}\right\}$ and $\left\{y_{n}\right\}$ such that $x_{n}=\frac{1}{2}-\frac{1}{n}$ and $y_{n}=\frac{1}{2}$, for all $n>1$. Clearly $\lim _{n} f x_{n}=$ $\lim _{n} f y_{n}=\frac{1}{2} \in\left\{\frac{1}{2}\right\}=\lim _{n} T x_{n}=\lim _{n} T y_{n}$ and $\frac{1}{2} \in f X$. Hence $f$ is strongly tangential with respect to $T$. The point $z=\frac{1}{2}$ is a strict coincidence point and $f f \frac{1}{2}=f \frac{1}{2}$, i.e., $(f, T)$ is coincidentally idempotent.

For $x, y \in\left[0, \frac{1}{2}\right]$, we have:

$$
\delta(T x, T y)=0 \leq \frac{1}{3} d(f x, T x) .
$$

For $x \in\left[0, \frac{1}{2}\right]$ and $y \in\left(\frac{1}{2}, 5\right]$, we have:

$$
\delta(T x, T y)=1 \leq \frac{7}{6}=\frac{1}{3} d(f y, T y) .
$$

For $x, y \in\left(\frac{1}{2}, 5\right]$, we have:

$$
\delta(T x, T y)=\frac{1}{2} \leq \frac{7}{6}=\frac{1}{3} d(f x, T x) .
$$

For $x \in(1 / 2,5]$ and $y \in[0,1 / 2]$, we have:

$$
\delta(T x, T y)=1 \leq \frac{7}{6}=\frac{1}{3} d(f y, T y) .
$$

Thus $f$ and $T$ satisfy Corollary 3.1 for $\alpha=\frac{1}{3} \in\left[0, \frac{1}{2}\right)$. Also $T \frac{1}{2}=\left\{f \frac{1}{2}\right\}=\left\{\frac{1}{2}\right\}$, i.e., $\frac{1}{2}$ is the unique common strict fixed point of $f$ and $T$. It is interesting to point out here that both $f$ and $T$ are discontinuous mappings and $f X \nsubseteq T X$.

Now we establish coincidence and common fixed point for two hybrid pairs of mappings satisfying Kannan type contraction using Hausdorff distance.

Theorem 3.2. Let $f, g: X \rightarrow X$ be single valued mappings and $S, T: X \rightarrow C B(X)$ be multivalued mappings of metric space $(X, d)$. If there exists $\alpha \in\left[0, \frac{1}{2}\right)$ such that

$$
H(S x, T y) \leq \alpha[d(f x, S x)+d(g y, T y)], \forall x, y \in X .
$$

and pair $(f, g)$ is strongly tangential with respect to $(S, T)$. Then pairs $(f, S)$ and $(g, T)$ have a coincidence point. Moreover, $f, g, S$ and $T$ have a common fixed point if hybrid pairs $(f, S)$ and $(g, T)$ are coincidentally idempotent.

Proof. Suppose that $(f, g)$ is strongly tangential with respect to $(S, T)$. Hence, there exist sequences $\left\{x_{n}\right\}$ and $\left\{y_{n}\right\}$ in $X$ such that $\lim _{n} f x_{n}=\lim _{n} g y_{n}=z \in A=\lim _{n} S x_{n}=\lim _{n} T y_{n}$ where $A \in C B(X), z \in X$ and $z \in f X \cap g X$. Hence there exist $u$ and $v \in X$ such that $f u=g v=z$.

Now we claim that $z=f u \in S u$, if not using $x=u$ and $y=y_{n}$ in condition (3.3),

$$
H\left(S u, T y_{n}\right) \leq \alpha\left[d(f u, S u)+d\left(g y_{n}, T y_{n}\right)\right]
$$

Taking limit as $n \rightarrow \infty$, we get

$$
H(S u, A) \leq \alpha d(f u, S u)+\alpha d(z, A)
$$

or

$$
H(S u, A) \leq \alpha d(f u, S u)
$$

But $f u=z \in A$. So,

$$
d(f u, S u) \leq \alpha d(f u, S u)<d(f u, S u),
$$

a contradiction. Therefore, $f u \in S u$. Hence, $f$ and $S$ have a coincidence point.

Now we claim that $z=g v \in T v$, if not using $x=x_{n}$ and $y=v$ in condition (3.3),

$$
H\left(S x_{n}, T v\right) \leq \alpha d\left(f x_{n}, S x_{n}\right)+\alpha d(g v, T v) .
$$


Taking limit as $n \rightarrow \infty$, we get

$$
H(A, T v) \leq \alpha d(z, A)+\alpha d(g v, T v)
$$

or

$$
H(A, T v) \leq \alpha d(g v, T v)
$$

But $g v=z \in A$. So,

$$
d(g v, T v) \leq \alpha d(g v, T v)<d(g v, T v),
$$

a contradiction. Therefore, $g v \in T v$. Hence, $g$ and $T$ have a coincidence point. Hence $z \in S u=T v=\{z\}$. Now since $(f, S)$ is coincidentally idempotent, $f u \in S u$ implies $f f u=f u \in S u$.

Now we claim that $z=f z \in S z$, if not using $x=z$ and $y=y_{n}$ in condition (3.3),

$$
H\left(S z, T y_{n}\right) \leq \alpha d(f z, S z)+\alpha d\left(g y_{n}, T y_{n}\right) .
$$

Taking limit as $n \rightarrow \infty$, we get

$$
H(S z, A) \leq \alpha d(f z, S z)+\alpha d(z, A)
$$

or

$$
H(S z, A) \leq \alpha d(f z, S z)
$$

But $f z=z \in A$, i.e.,

$$
d(f z, S z) \leq \alpha d(f z, S z)<d(f z, S z),
$$

a contradiction. Therefore, $f z \in S z$. Similarly, $(g, T)$ is coincidentally idempotent, $g v \in T v$ implies $g g v=g v \in T v$. Now we claim that $z=g z \in T z$, if not using $x=x_{n}$ and $y=z$ in condition (3.3),

$$
H\left(S x_{n}, T z\right) \leq \alpha d\left(f x_{n}, S x_{n}\right)+\alpha d(g z, T z) .
$$

Taking limit as $n \rightarrow \infty$, we get

$$
H(A, T z) \leq \alpha d(z, A)+\alpha d(g z, T z)
$$

or

$$
H(A, T z) \leq \alpha d(g z, T z)
$$

But $g z=z \in A$, so,

$$
d(g z, T z) \leq \alpha d(g z, T z)<d(g z, T z)
$$

a contradiction. Therefore, $g z \in T z$.

Therefore $z$ is a common fixed point of $f, g, T$ and $S$.

Taking $f=g$ and $T=S$ in Theorem 3.2, we obtain the following corollary:

Corollary 3.2. Let $f: X \rightarrow X$ be a single valued mapping and $T: X \rightarrow C B(X)$ be a multivalued mapping of metric space $(X, d)$. If there exists $\alpha \in\left[0, \frac{1}{2}\right)$ such that

$$
H(T x, T y) \leq \alpha[d(f x, T x)+d(f y, T y)], \forall x, y \in X
$$

and $f$ is strongly tangential with respect to $T$. Then $f$ and $T$ have a coincidence point. Moreover, $f$ and $T$ have a common fixed point if hybrid pair $(f, T)$ is coincidentally idempotent.

Example 3.2. Let $X=[0,12]$ and $d$ be the usual metric on $X$. Let a hybrid pair of mappings $(f, T)$ on $X$ be defined as follows:

$$
f x=\left\{\begin{array}{ll}
4-x, & 0 \leq x \leq 2 \\
12, & 2<x \leq 12,
\end{array} \quad T x= \begin{cases}{[2,3],} & 0 \leq x \leq 2 \\
{\left[1, \frac{3}{2}\right],} & 2<x \leq 12\end{cases}\right.
$$

Consider two sequences $\left\{x_{n}\right\}$ and $\left\{y_{n}\right\}$ such that $x_{n}=2-\frac{1}{n}$ and $y_{n}=2$, for all $n>1$, Clearly $\lim _{n} f x_{n}=$ $\lim _{n} f y_{n}=2 \in \lim _{n} T x_{n}=\lim _{n} T y_{n}$ and $2 \in f X$. Hence $f$ is strongly tangential with respect to $T$. The point $z=2$ is a coincidence point and $f f 2=f 2$, i.e., $(f, T)$ is coincidentally idempotent.

For $x, y \in[0,2]$, we have:

$$
H(T x, T y)=0 \leq \frac{2}{21} d(f y, T y) .
$$


For $x \in[0,2]$ and $y \in(2,12]$, we have:

$$
H(T x, T y)=1 \leq \frac{2}{21} d(f y, T y)
$$

For $x, y \in(2,12]$, we have:

$$
H(S x, T y)=0 \leq \frac{2}{21} d(f x, T x)
$$

For $x \in(2,12]$ and $y \in[0,2]$, we have

$$
H(T x, T y)=1 \leq \frac{2}{21} \cdot d(f x, T x)
$$

Thus $f$ and $T$ satisfy Corollary 3.2 for $\alpha=\frac{2}{21} \in\left[0, \frac{1}{2}\right)$. Also $f 2=2 \in[2,3]=S 2$, i.e., 2 is the common fixed point of $f$ and $T$. It is interesting to point out here that both $f$ and $T$ are discontinuous mappings and $f X \nsubseteq T X$.

\section{Application}

In recent years, a lot of work has been done wherein solutions and common solutions of some functional equations, including systems of functional equations in dynamic programming are obtained using suitable fixed /common fixed point results. The existence of solutions of functional equation arising in dynamic programming was first studied by Bellman [2] and in 1978 Bellman and Lee [3] stated the basic form of the functional equations. The purpose of this section is to prove the existence of solutions for a system of functional equations arising in dynamic programming using Theorem 3.1.

Let $B(W)$ be the set of all closed and bounded real-valued functions on $W$. For an arbitrary $h, k \in B(W)$ define $\|h\|=\sup _{x \in W}|h(x)|,\|k\|=\sup _{x \in W}|k(x)|$ and $\delta(h, k)=\sup _{x \in W}|h(x)-k(x)|$. Also, $(B(W),\|\cdot\|)$ is a Banach space wherein convergence is uniform.

We consider the operators $T_{i}, A_{i}: B(W) \rightarrow B(W)$ given by

$$
\begin{cases}T_{i} h(x)=\sup _{y \in D}\left\{g(x, y)+G_{i}(x, y, h(\tau(x, y))\},\right. & \mathrm{i}=1,2, \\ A_{i} k(x)=\sup _{y \in W}\{\dot{g}(x, y)+G i(x, y, k(\tau(x, y))\}, & \mathrm{i}=1,2\end{cases}
$$

for $h, k \in B(W)$, where $\tau: W \times D \rightarrow W, g, g^{\prime}: W \times D \rightarrow \mathbb{R}, G_{i}, G_{i}^{\prime}: W \times D \times \mathbb{R} \rightarrow \mathbb{R}$ are given mappings, while $W \in U$ is a state space, $D \in V$ is a decision space and $U, V$ are Banach spaces. These mappings are well-defined if the functions $g_{i}, \dot{g}_{i}, G_{i}$ and $G_{i}$ are bounded. Also, denote

$$
\Theta(h, k)=\alpha\left\{d\left(A_{1} h, T_{1} h\right)+d\left(A_{2} k, T_{2} k\right)\right\},
$$

for $\alpha=\left[0, \frac{1}{2}\right)$ and $h, k \in B(W)$.

Theorem 4.1. Let $T_{i}, A_{i}: B(W) \rightarrow B(W)$ be given by 4.1 , for $i=1,2$. Suppose that the following hypotheses hold:

1. $\mid G_{1}\left(x, y, h(\tau(x, y))-G_{2}(x, y, k(\tau(x, y))) \mid \leq \Theta(h, k)\right.$, for all $x \in W, y \in D$,

2. $g, g^{\prime}: W \times D \rightarrow \mathbb{R}$ and $G_{i}, G_{i}^{\prime}: W \times D \times \mathbb{R} \rightarrow \mathbb{R}$ are bounded functions, for $i=1,2$;

3. There exists sequences $\left\{h_{n}\right\},\left\{k_{n}\right\} \in B(W)$ and functions $h^{*} \in B(W)$ such that $\lim _{n \rightarrow \infty} T_{1} h_{n}=\lim _{n \rightarrow \infty} T_{2} k_{n}=A \in B(W)$ and $\lim _{n \rightarrow \infty} A_{1} h_{n}=\lim _{n \rightarrow \infty} A_{2} k_{n}=h^{*} \in A$ and $h^{*} \in A_{1} \cap A_{2} ;$

4. $A_{1} A_{1} h=A_{1} h$, whenever $A_{1} h \in T_{1} h$ and $A_{2} A_{2} k=A_{2} k$, whenever $A_{2} k \in T_{2} k$ for some $h, k \in B(W)$.

Then the system of functional equations (4.1) has a bounded solution. 
Proof. Consider the $\delta(h, k)=\sup \{|h(x)-k(x)|\}$ for any $h, k \in B(W)$. Let $\lambda$ be an arbitrary positive number, $x \in W$. Then there exists $y_{1}, y_{2} \in D$ such that

$$
\begin{aligned}
& T_{1} h(x)<g\left(x, y_{1}\right)+G_{1}\left(x, y_{1}, h\left(\tau\left(x, y_{1}\right)\right)\right)+\lambda, \\
& T_{2} k(x)<g\left(x, y_{2}\right)+G_{2}\left(x, y_{2}, k\left(\tau\left(x, y_{2}\right)\right)\right)+\lambda .
\end{aligned}
$$

Also by definition

$$
\begin{aligned}
& T_{1} h(x) \geq g\left(x, y_{2}\right)+G_{1}\left(x, y_{2}, h\left(\tau\left(x, y_{2}\right)\right)\right) \\
& T_{2} k(x) \geq g\left(x, y_{1}\right)+G_{2}\left(x, y_{1}, k\left(\tau\left(x, y_{1}\right)\right)\right)
\end{aligned}
$$

Next, by using (4.2) and (4.5) we obtain

$$
\begin{aligned}
T_{1} h(x)-T_{2} k(x) & <G_{1}\left(x, y_{1}, h\left(\tau\left(x, y_{1}\right)\right)\right)-G_{2}\left(x, y_{1}, k\left(\tau\left(x, y_{1}\right)\right)\right)+\lambda \\
& \leq\left|G_{1}\left(x, y_{1}, h\left(\tau\left(x, y_{1}\right)\right)\right)-G_{2}\left(x, y_{1}, k\left(\tau\left(x, y_{1}\right)\right)\right)\right|+\lambda \\
& \leq \Theta(h, k)+\lambda
\end{aligned}
$$

Also from (4.3) and (4.4)

$$
T_{2} k(x)-T_{1} h(x)<\Theta(h, k)+\lambda
$$

Combining, we get

$$
\left|T_{1} h(x)-T_{2} k(x)\right|<\Theta(h, k)+\lambda .
$$

Implying thereby

$$
\delta\left(T_{1} h, T_{2} k\right) \leq \Theta(h, k)+\lambda .
$$

Notice that, the last inequality does not depend on $x \in W$ and $\lambda>0$ is taken arbitrarily.

Therefore we obtain

$$
\delta\left(T_{1} h, T_{2} k\right) \leq \Theta(h, k) .
$$

for each $t \in(0, \infty)$. Also by hypothesis (3), $\left(A_{1}, A_{2}\right)$ is strongly tangential with respect to $\left(T_{1}, T_{2}\right)$. Thus using condition (4) and taking $f=A_{1}, S=T_{1}, g=A_{2}, T=T_{2}$ all the hypotheses of Theorem 3.1 are satisfied for $\alpha=\left[0, \frac{1}{2}\right)$. Hence using Theorem 3.1, the mapping $T_{1}, T_{2}, A_{1}$ and $A_{2}$ have a unique common fixed point, i.e., the system of functional equations (4.1) has a unique bounded solution.

\section{References}

[1] Banach S., Sur les operations dans les ensembles abstrait set leur application aux equations integrals, Fund. Math., 3(1922), 133-181.

[2] Bellman, R., Methods of Nonlinear Analysis, Vol II, Academic Press, New York, 1973.

[3] Bellman, R., Lee E. S., Functional equations in dynamic programming, Aequationes Math., 17(1) (1978), 1-18.

[4] Chauhan, S., Imdad M. , Karapinar E. and B. Fisher, An integral type fixed point theorem for multi-valued mappings employing strongly tangential property, J. Egyptian Math. Soc., 22 (2) (2014), 258-264.

[5] Imdad, M., Ahmad,A., Kumar, S. On nonlinear nonself hybrid contractions, Radovi Matematicki. 10(2) (2001), 233-244.

[6] Kaneko, H., A common fixed point of weakly commuting multivalued mappings, Math. Japon., 33(5) (1988), 741-744.

[7] Kannan, R., Some results on fixed points, Bull. Calcutta Math. Soc., 60(1968), 71-76.

[8] Kannan, R., Some results on fixed points. II, American Mathematical Monthly, 76, (1969), 405-408.

[9] Markin, J., A fixed point theorem for set-valued mappings, Bull. Amer. Math. Soc. 74 (1968), 639-640.

[10] Nadler, Jr. S. B., Multl-valued contraction mappings, Pacific J. Math. 30(1969), 475-486. 
[11] Seesa, S., On a weak commutativity condition of mappings in Fixed point considerations, Publ. Inst. Math. 32 (46), (1982),149-153.

[12] Sintunavarat, W., Kumam, P., Gregus-type common fixed point theorems for tangential multi-valued mappings of integral type in metric spaces, Int. J. Math. Math. Sci. 12 (2011). Article ID 923458.

[13] Subrahmanyam, V., Completeness and fixed-points, Monatsh. Math. 80, (1975), 325-330 .

\section{Affiliations}

ANITA TOMAR

ADDRESS: Government P.G. College Dakpathar Dehradun (Uttarakhand), India.

E-MAIL: anitatmr@yahoo.com

SHIVANGI UPADHYAY

ADDRESS: Government P.G. College Dakpathar Dehradun (Uttarakhand), India. E-MAIL: shivangiupadhyay90@gmail.com

RITU SHARMA

ADDRESS: Government P.G. College Dakpathar Dehradun (Uttarakhand), India.

E-MAIL: ritus4184@gmail.com 